Issued by Sandia National Laboratories, operated for the United States Department of Energy by Sandia Corporation.

NOTICE: This report was prepared as an account of work sponsored by an agency of the United States Government. Neither the United States Government, nor any agency thereof, nor any of their employees, nor any of their contractors, subcontractors, or their employees, make any warranty, express or implied, or assume any legal liability or responsibility for the accuracy, completeness, or usefulness of any information, apparatus, product, or process disclosed, or represent that its use would not infringe privately owned rights. Reference herein to any specific commercial product, process, or service by trade name, trademark, manufacturer, or otherwise, does not necessarily constitute or imply its endorsement, recommendation, or favoring by the United States Government, any agency thereof, or any of their contractors or subcontractors. The views and opinions expressed herein do not necessarily state or reflect those of the United States Government, any agency thereof, or any of their contractors.

Printed in the United States of America. This report has been reproduced directly from the best available copy.

Available to DOE and DOE contractors from

Office of Scientific and Technical Information

P.O. Box 62

Oak Ridge, TN 37831

Prices available from (703) 605-6000

Web site: http://www.ntis.gov/ordering.htm

Available to the public from

National Technical Information Service

U.S. Department of Commerce

5285 Port Royal Rd

Springfield, VA 22161

NTIS price codes

Printed copy: A03

Microfiche copy: A01

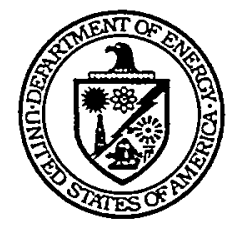




\section{DISCLAIMER}

Portions of this document may be illegible in electronic image products. Images are produced from the best available original document. 
SAND99-8249

Unlimited Release

Printed June 1999

\title{
Hydrodynamic Dispersion of a Neutral Non-Reacting Solute in Electroosmotic Flow
}

\author{
Stewart K. Griffiths and Robert H. Nilson \\ Sandia National Laboratories \\ Livermore, California 94551-0969
}

\begin{abstract}
Analytical methods are employed to determine the axial dispersion of a neutral non-reacting solute in an incompressible electroosmotic flow. In contrast to previous approaches, the dispersion is obtained here by solving the time-dependent diffusion-advection equation in transformed spatial and temporal coordinates to obtain the two-dimensional late-time concentration field. The coefficient of dispersion arises as a separation eigenvalue, and its value is obtained as a necessary condition for satisfying all of the required boundary conditions. Solutions based on the Debye-Hückel approximation are presented for both a circular tube and a channel of infinite width. These results recover the well-known solutions for dispersion in pressure-driven flows when the Debye length is very large. In this limit, the axial dispersion is proportional to the square of the Peclet number based on the characteristic transverse dimension of the tube or channel. In the limit of very small Debye lengths, we find that the dispersion varies as the square of the Peclet number based on the Debye length. Simple approximations to the coefficient of dispersion as a function of the Debye length and Peclet number are also presented.
\end{abstract}

\section{INTRODUCTION}

Microchannel devices are finding increased use in the separation, identification and synthesis of a wide range of chemical and biological species. Employing transverse channel dimensions in the range from a few microns to about one millimeter, such systems may permit the miniaturization and largescale integration of many chemical processes in a manner analogous to that already achieved in microelectronics. Applications for microchannel devices now under development include such diverse processes as DNA sequencing, immunoassay, the identification of explosives, identification of chemical and biological warfare agents, and the synthesis of chemicals and drugs.

Electroosmotic flows $[1,2]$ offer two important benefits over pressure-driven flows for the small physical dimensions characteristic of microchannel devices. First, fluid speeds in electroosmotic flows are independent of the transverse dimension of the tube or channel over a wide range of conditions, making this technique for driving fluid motion extensible to extremely small physical scales. In contrast, pressure-driven flows require a pressure gradient that increases inversely with the square of the minimum transverse dimension to maintain a given fluid speed. Second, the profile of the fluid velocity across a tube or channel is essentially flat, again over a very wide range of conditions. All variations in the axial velocity are confined to a small region, comparable in thickness to the electric Debye layer, adjacent to the tube or channel walls. The benefit of this flat velocity profile is that samples may be transported over long ranges with very little dispersion due to nonuniform fluid speeds.

To help understand hydrodynamic dispersion in electroosmotic flow, we will consider the diffusive 
and advective transport of a neutral non-reacting solute within a tube or wide channel, as shown in Fig. 1. In either case, the transverse dimension, $2 a$, is assumed to be small compared to the tube or channel length. An electric field applied along the axis in the $x$ direction produces an electroosmotic flow, and the solute carried by this flow is spread in the axial direction by diffusion and dispersion. This is illustrated by the Gaussian profile indicating the axial variation of the local transverse average of the concentration field sometime following the introduction of an instantaneous planar source. Analogous spreading occurs at a translating solute interface, such that an initially discontinuous step in the solute concentration broadens with increasing time.

As solute is convected by the electroosmotic flow, axial diffusion tends to spread the profile or, alternatively, to smear the boundary in the case of a moving interface. At the same time, the nonuniform profile of the fluid velocity leads to transverse variations in the concentration field, giving rise to a transverse diffusive flux. At sufficiently late times, advective transport in the axial direction is just balanced by diffusive transport in the transverse direction. The resulting hydrodynamic dispersion produces a mean axial concentration profile consistent with diffusive transport alone, though the apparent diffusivity may be much larger than the true molecular diffusivity and generally depends on the Peclet number.

\section{GOVERNING EQUATIONS}

Assuming that the fluid is incompressible and that transport properties are constant, the timedependent concentration field is governed by

$$
\frac{\partial c}{\partial t}+\mathbf{u} \cdot \nabla c=D \nabla^{2} c
$$

where $c$ is the local solute concentration, $t$ is time, $\mathrm{u}=u \mathbf{i}+v \mathbf{j}$ is the local fluid velocity, and $D$ is the coefficient of diffusion. Further assuming that the flow is steady and that inertial effects are small, the momentum equation may be written as

$$
\mu \nabla^{2} \mathrm{u}=\rho_{e} \nabla \phi
$$

where $\mu$ is the fluid viscosity, $\rho_{e}$ is the net local charge density, and $\phi$ is the local electric potential. Finally, for a dielectric constant, $\epsilon$, that does not vary with position, the Poisson equation governing the electric field is

$$
\epsilon \nabla^{2} \phi=-\rho_{e}
$$

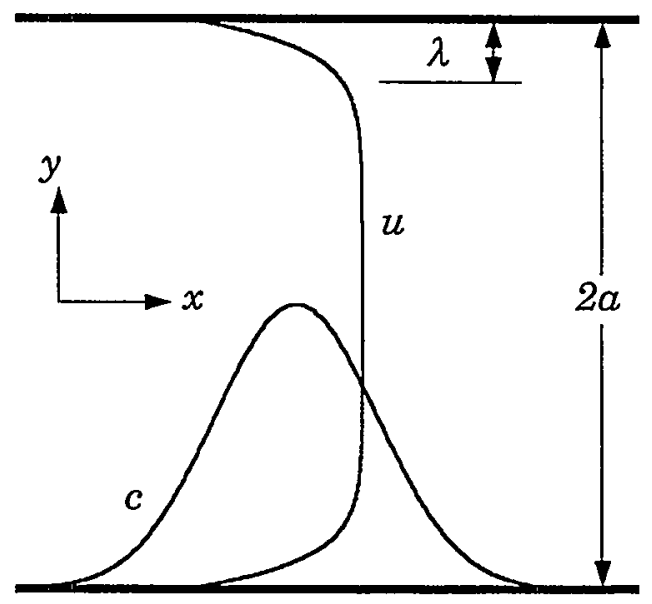

Figure 1. Schematic cross-section of a tube or channel. Spreading of an initial concentration pulse results from diffusion and dispersion. Fluid velocities in an electroosmotic flow are zero on the solid boundaries and exhibit a boundary layer thickness comparable to the Debye length.

and the local charge density may be related to the electric potential through the Boltzmann distribution given by $\rho_{e}=-2 F z c_{e} \sinh (z F \phi / R T)$ for equivalent ions, where $F$ is the Faraday constant, $z$ is the ion charge number, $c_{e}$ is the bulk fluid ion concentration, $R$ is the universal gas constant, and $T$ is the temperature.

To solve generally for the concentration field, we now introduce a set of dimensionless variables. The new normalized dependent variables are taken as $c^{*}=c / c_{o}, \mathrm{u}^{*}=\mathrm{u} / U$ and $\phi^{*}=\phi / \zeta$, where $c_{o}$ is some reference concentration yet to be specified, $U$ is the mean axial fluid speed spatially averaged across the tube or channel, and $\zeta$ is the electric potential on the tube or channel wall. The new independent variables are $x^{*}=(x-U t) / a, y^{*}=y / a$ and $t^{*}=D t / a^{2}$, where $x$ and $y$ are the axial and transverse coordinates, and $a$ is the tube radius or channel half-height. This normalization leads to three new parameters, the normalized Debye length,

$$
\lambda^{* 2}=\frac{1}{\kappa^{* 2}}=\left(\frac{\lambda}{a}\right)^{2}=\frac{\epsilon R T}{2 F^{2} z^{2} c_{e} a^{2}}
$$

the normalized wall potential, $\zeta^{*}=z F \zeta / R T$, and the Peclet number, $P e=U a / D$ indicating the relative importance on advective and diffusive transport. 
Introducing these normalized variables into the primitive governing equations and rearranging slightly then yields

$$
\begin{gathered}
\frac{\partial c^{*}}{\partial t^{*}}+P e\left(\mathbf{u}^{*} \cdot \nabla c^{*}-\frac{\partial c^{*}}{\partial x^{*}}\right)=\nabla^{2} c^{*} \\
\beta \nabla^{2} \mathbf{u}^{*}=-\nabla^{2} \phi^{*} \mathbf{E}^{*}
\end{gathered}
$$

and

$$
\lambda^{* 2} \nabla^{2} \phi^{*}=\frac{1}{\zeta^{*}} \sinh \left(\zeta^{*} \phi^{*}\right)
$$

for Eqs. (1), (2) and (3), respectively. The new dependent variable, $\mathrm{E}^{*}=-\nabla \phi / E_{x}$ is the local electric field vector normalized by the applied axial electric field, $E_{x}$. Note that the operators $\nabla$ and $\nabla^{2}$ above are implicitly considered to involve derivatives with respect to the normalized independent variables $x^{*}$ and $y^{*}$ when applied to any normalized dependent variable.

The normalization additionally introduces one dimensionless unknown constant, $\beta=-\mu U / \epsilon \zeta E_{x}$. This constant is the ratio of the mean axial fluid speed to the Helmholtz-Smoluchowski speed for flow past a plane charged surface. Its value is given by the condition

$$
(n+1) \int_{0}^{1} u^{*} y^{* n} d y^{*}=1
$$

in accordance with the definition of the mean fluid speed, $U$. The parameter $n$ in Eq. (8) is used to describe either the planer or axisymmetric geometries by taking $n=0$ or 1 , respectively.

Recognizing that the incompressible flow field in a long tube or channel must be one dimensional and that the radial component of the fluid velocity is therefore everywhere zero, the diffusion-advection equation (5) may be rewritten as

$$
\begin{aligned}
\frac{\partial c^{*}}{\partial t^{*}}+P e & \left(u^{*}-1\right) \frac{\partial c^{*}}{\partial x^{*}} \\
= & \frac{\partial^{2} c^{*}}{\partial x^{* 2}}+\frac{1}{y^{* n}} \frac{\partial}{\partial y^{*}}\left(y^{* n} \frac{\partial c^{*}}{\partial y^{*}}\right)
\end{aligned}
$$

where $u^{*}$ is the normalized local fluid speed in the axial direction.

Boundary conditions for the normalized concentration are $c^{*} \rightarrow 1$ as $x^{*} \rightarrow-\infty$ and $c^{*} \rightarrow 0$ as $x^{*} \rightarrow$ $\infty$ for the case of a translating interface, and $c^{*} \rightarrow 0$ as $x^{*} \rightarrow \pm \infty$ for the instantaneous plane source. The initial conditions are $c^{*}\left(x^{*}, y^{*}, t^{*}\right)=\hat{c}^{*}\left(x^{*}\right)$ at $t^{*}=0$, where

$$
\hat{c}^{*}=\frac{1}{2} \operatorname{erfc} \frac{x^{*}}{2 \sqrt{t^{*}}} \quad \text { as } \quad t^{*} \rightarrow 0
$$

for the interface, and

$$
\hat{c}^{*}=\frac{e^{-x^{* 2} / 4 t^{*}}}{2 \sqrt{\pi t^{*}}} \quad \text { as } \quad t^{*} \rightarrow 0
$$

for the plane source. The first of these approximates an initial unit step at $x^{*}=0$, while the second approximates an initial plane source of unit strength centered at $x^{*}=0$. Since we are interested here in a late-time solution, it is convenient to express the initial conditions in terms of moments of the solute distribution. That is,

$$
\int_{\infty}^{\infty} x^{* p} \int_{0}^{1}\left(c^{*}-\hat{c}^{*}\right) y^{* n} d y^{*} d x^{*}=0
$$

as $t^{*} \rightarrow 0$, for all $p \geq 0$. If all moments of the functions $c^{*}\left(x^{*}, y^{*}, t^{*} \rightarrow 0\right)$ and $\hat{c}^{*}\left(x^{*}\right)$ are equal, then the two functions are the same.

Now recognizing that the second derivative of the electric potential in the axial direction is small compared to that in the transverse direction and that the normalized electric field is defined such that $\mathbf{E}^{*} \cdot \mathbf{i}=1$, the axial component of the momentum equation (6) may be written as

$$
\frac{\beta}{y^{* n}} \frac{d}{d y^{*}}\left(y^{* n} \frac{d u^{*}}{d y^{*}}\right)=-\frac{1}{y^{* n}} \frac{\partial}{\partial y^{*}}\left(y^{* n} \frac{\partial \phi^{*}}{\partial y^{*}}\right)
$$

and the Poisson-Boltzmann equation becomes

$$
\frac{\lambda^{* 2}}{y^{* n}} \frac{\partial}{\partial y^{*}}\left(y^{* n} \frac{\partial \phi^{*}}{\partial y^{*}}\right)=\frac{1}{\zeta^{*}} \sinh \left(\zeta^{*} \phi^{*}\right) \approx \phi^{*}
$$

The far right-hand portion of this expression represents the Debye-Hückel approximation for the case in which $\zeta^{*}$ is small.

Boundary conditions for the fluid velocities are $d u^{*} / d y^{*}=0$ at $y^{*}=0$ and $u^{*}=0$ at $y^{*}=1$. Those for the electric potential are $\partial \phi^{*} / \partial y^{*}=0$ at $y^{*}=0$ and $\phi^{*}=1$ at $y^{*}=1$. The resulting axial fluid speed in the Debye-Hückel limit is

$$
u^{*}=\frac{\cosh \left(y^{*} / \lambda^{*}\right)-\cosh \left(1 / \lambda^{*}\right)}{\lambda^{*} \sinh \left(1 / \lambda^{*}\right)-\cosh \left(1 / \lambda^{*}\right)}
$$

for a channel, while that for a tube is

$$
u^{*}=\frac{I_{0}\left(y^{*} / \lambda^{*}\right)-I_{0}\left(1 / \lambda^{*}\right)}{2 \lambda^{*} I_{1}\left(1 / \lambda^{*}\right)-I_{0}\left(1 / \lambda^{*}\right)}
$$

The corresponding values of $\beta$ used to obtain these results are $\beta=1-\lambda^{*} \tanh \left(1 / \lambda^{*}\right)$ and $\beta=$ $1-2 \lambda^{*} I_{1}\left(1 / \lambda^{*}\right) / I_{0}\left(1 / \lambda^{*}\right)=I_{2}\left(1 / \lambda^{*}\right) / I_{0}\left(1 / \lambda^{*}\right)$ for a 
channel and tube, respectively. The result above for a tube is exactly that previously obtained by Rice and Whitehead [2].

\section{METHOD OF SOLUTION}

To solve Eq. (9) we now introduce one more transformation of the axial coordinate, $\eta=x^{*} / 2 \sqrt{t^{*}}$. Introducing this new variable into the diffusionadvection equation yields

$$
\begin{array}{rl}
\frac{\partial^{2} c^{*}}{\partial \eta^{2}}+2 & 2 \eta \frac{\partial c^{*}}{\partial \eta}=2 \sqrt{t^{*}} P e\left(u^{*}-1\right) \frac{\partial c^{*}}{\partial \eta} \\
+4 t^{*} & {\left[\frac{\partial c^{*}}{\partial t^{*}}-\frac{1}{y^{* n}} \frac{\partial}{\partial y^{*}}\left(y^{* n} \frac{\partial c^{*}}{\partial y^{*}}\right)\right]}
\end{array}
$$

Note that the left side of Eq. (17) yields a homogeneous solution of the form $c^{*} \propto \operatorname{erfc} \eta$. This is the well known solution for a translating interface with either no flow, $P e \rightarrow 0$, or a uniform axial fluid speed, $u^{*}-1=0$. Under the same restrictions, solutions for the case of an instantaneous plane source are obtained from the left of Eq. (17) along with the term $4 t^{*} \partial c^{*} / \partial t^{*}$ on the right. The solution in this case has the form $c^{*} \propto e^{-\eta^{2}} / \sqrt{t^{*}}$.

Based on Taylor's observation [3] that the transverse variation in solute concentration is proportional to the axial concentration gradient, we now seek a solution to Eq. (17) in the form

where

$$
c^{*} t^{* m / 2}=f+\sum_{j=1}^{\infty} \frac{P e^{j}}{2^{j} t^{* j / 2}} \frac{d^{j} f}{d \eta^{j}} g_{j}
$$

$$
f=f_{0}+\sum_{k=1}^{\infty} \frac{f_{k}}{2^{k} t^{* k / 2}}
$$

The functions $g_{j}$ depend only on the transverse position, $y^{*}$, while the functions $f_{k}$ depend only on the transformed axial position, $\eta$. Without loss of generality, we assume that the spatial average of all $g_{j}$ over the tube or channel cross-section is zero such that the axial variation of the mean concentration field is given by the function $f$ alone. Values of the parameter $m=0$ or $m=1$ identify the case of an translating interface or instantaneous source, respectively.

Boundary conditions for the functions $f_{k}$ are $f_{0} \rightarrow 1$ as $\eta \rightarrow-\infty$ and $f_{0} \rightarrow 0$ as $\eta \rightarrow \infty$ for the case of a translating interface, and $f_{0} \rightarrow 0$ as $\eta \rightarrow \pm \infty$ for the instantaneous plane source. For this latter case, the initial condition (12) requires that

$$
\int_{-\infty}^{\infty} 2 f_{0} d \eta=1 \quad m=1
$$

Boundary conditions for higher-order functions for both cases are $f_{k} \rightarrow 0$ as $\eta \rightarrow \pm \infty$. The initial condition (12) additionally requires that the higher-order functions $f_{k}$ for both the translating interface, $m=0$ and instantaneous plane source, $m=1$, must also satisfy

$$
\int_{-\infty}^{\infty} \eta^{p} f_{k} d \eta=0 \quad \text { for } \quad 0 \leq p \leq k+m-1
$$

for all $k \geq 1$. The higher-order moments of $f_{k}$ need not vanish since the net exponent of the time in the corresponding terms of Eq. (12) is greater than zero for $p>k+m-1$.

Boundary conditions for the functions $g_{j}$ are $d g_{j} / d y^{*}=0$ at $y^{*}=0$ and $y^{*}=1$. Based on the condition that all axial variation of the concentration field is carried by the function $f$ alone, we additionally require that

$$
\int_{0}^{1} g_{j} y^{* n} d y^{*}=0
$$

for all $j$. Because of this, we see that the timedependent function $f$ given by Eq. (18b) represents the local transverse spatial average of the solute concentration as a function of the transformed axial position and that all axial dispersion will be evident in this function alone.

Now substituting Eqs. (18a) and (18b) into Eq. (17) and grouping like powers of time yields

$$
2 P e \frac{d f_{0}}{d \eta}\left[\frac{1}{y^{* n}} \frac{d}{d y^{*}}\left(y^{* n} \frac{d g_{1}}{d y^{*}}\right)-\left(u^{*}-1\right)\right]=0
$$

for the order $\sqrt{t^{*}}$ term. Again grouping like powers, the order one terms yield

$$
\begin{gathered}
\llbracket 1+\left[\frac{1}{y^{* n}} \frac{d}{d y^{*}}\left(y^{* n} \frac{d g_{2}}{d y^{*}}\right)-\left(u^{*}-1\right) g_{1}\right] P e^{2} \rrbracket \frac{d^{2} f_{0}}{d \eta^{2}} \\
+2 \eta \frac{d f_{0}}{\partial \eta}+2 m f_{0}=0
\end{gathered}
$$

Again, $m=0$ for the case of a translating interface and $m=1$ for the case of an instantaneous plane source.

Noting that $d f_{0} / d \eta$ cannot be everywhere zero, Eq. (22) requires that

$$
g_{1}^{\prime \prime}+n \frac{g_{1}^{\prime}}{y^{*}}-\left(u^{*}-1\right)=0
$$

where primes denote differentiation of the functions $g_{j}$ with respect to $y^{*}$; when later applied to the functions $f_{k}$ these primes denote differentiation with respect to $\eta$. Further noting that $f_{0}$ is a function of $\eta$ 
only, the leading factor on the left of Eq. (23) must not depend on $y^{*}$. Thus necessary conditions for the existence of a solution in the form of Eq. (18) are

$$
\begin{gathered}
g_{2}^{\prime \prime}+n \frac{g_{2}^{\prime}}{y^{*}}-\left(u^{*}-1\right) g_{1}=\alpha_{0} \\
\left(1+\alpha_{0} P e^{2}\right) f_{0}^{\prime \prime}+2 \eta f_{0}^{\prime}+2 m f_{0}=0
\end{gathered}
$$

where $\alpha_{0}$ is some yet unknown separation constant or eigenvalue.

Similarly, we find that the third-order function $g_{3}$ and first-order function $f_{1}$ must satisfy

$$
\begin{gathered}
g_{3}^{\prime \prime}+n \frac{g_{3}^{\prime}}{y^{*}}-\left(u^{*}-1\right) g_{2}-\alpha_{0} g_{1}=\alpha_{1} \\
\left(1+\alpha_{0} P e^{2}\right) f_{1}^{\prime \prime}+2 \eta f_{1}^{\prime}+2(m+1) f_{1}=-\alpha_{1} P e^{3} f_{0}^{\prime \prime \prime}
\end{gathered}
$$

Note that the form of Eq. (18) is critical to satisfying all of the necessary integral and boundary conditions on the functions $g_{j}$. If, for example, the function $f$ were taken as simply $f_{0}$, then only the single eigenvalue $\alpha_{0}$ ever appears in the problem. As a result, this simpler form of the solution can not satisfy both of the required conditions $g_{3}^{\prime}(0)=0$ and $g_{3}^{\prime}(1)=0$.

The functions $g_{j}$ are unusual in that they are governed by second-order equations but must satisfy two boundary conditions on their first derivatives. In general such a system of equations cannot be solved since only one constant of integration is available to satisfy the two boundary conditions. Here this problem is avoided by appropriate choices for the eigenvalues, $\alpha_{k}$. From the governing equar tion (24) and condition $g_{1}^{\prime}(0)=0$, the derivative $g_{1}^{\prime}(1)$ at the tube or channel wall can be written as

$$
g_{1}^{\prime}(1)=\int_{0}^{1}\left(u^{*}-1\right) y^{* n} d y^{*} \equiv 0
$$

Thus the first-order solution automatically satisfies $g_{1}^{\prime}(1)=0$ by the definition of $u^{*}$. For the higherorder terms, however, the conditions $g_{2}^{\prime}(1)=0$ and $g_{3}^{\prime}(1)=0$ require that

and $\alpha_{0}=-(n+1) \int_{0}^{1}\left[\left(u^{*}-1\right) g_{1}\right] y^{* n} d y^{*}$

$$
\alpha_{1}=-(n+1) \int_{0}^{1}\left[\left(u^{*}-1\right) g_{2}+\alpha_{0} g_{1}\right] y^{* n} d y^{*}
$$

respectively. The eigenvalues for this problem are thus uniquely determined by the two boundary conditions $g_{j}^{\prime}(0)=g_{j}^{\prime}(1)=0$. The two constants of integration for each function $g_{j}$ are then determined by the integral constraint (21) and either the condition $g_{j}^{\prime}(0)=0$ or $g_{j}^{\prime}(1)=0$.

\section{RESULTS}

Given the eigenvalues $\alpha_{0}$ and $\alpha_{1}$, Eqs. (26) and (28) can be solved to obtain the functions $f_{0}$ and $f_{1}$ describing axial variation of the solute concentration. For $m=0$, the case of a translating interface, these are

and

$$
f_{0}=\frac{1}{2} \operatorname{erfc} \eta^{\prime}
$$

$$
f_{1}=\frac{\alpha_{1} P e^{3}}{2 \sqrt{\pi\left(1+\alpha_{0} P e^{2}\right)^{3}}}\left(1-2 \eta^{2}\right) e^{-\eta^{2}}
$$

where

$$
\eta^{\prime}=\frac{\eta}{\sqrt{1+\alpha_{0} P e^{2}}}
$$

For $m=1$, the case of a instantaneous plane source of unit strength, the zeroth and first-order contributions are

and

$$
f_{0}=\frac{1}{2 \sqrt{\pi\left(1+\alpha_{0} P e^{2}\right)}} e^{-\eta^{\prime 2}}
$$

$$
f_{1}=\frac{\alpha_{1} P e^{3}}{2 \sqrt{\pi}\left(1+\alpha_{0} P e^{2}\right)^{2}}\left(3-2 \eta^{\prime 2}\right) \eta^{\prime} e^{-\eta^{\prime 2}}
$$

where $\eta^{\prime}$ is again given by Eq. (34). Details of the means by which these solutions were obtained are provided in the Appendix.

Noting the definition of $\eta$ given above, we see that Eqs. (32) and (35) describe a diffusion process in which the molecular diffusivity, $D$, is replaced by an effective diffusivity $D^{\prime}=D\left(1+\alpha_{0} P e^{2}\right)$. This observation confirms that late-time dispersion yields a mean axial concentration profile resembling that due to diffusion alone.

The coefficient of dispersion given by Eq. (30) is exactly that obtained by Taylor in his asymptotic analysis of dispersion in pressure-driven flows [3]. In that analysis, Taylor made an insightful observation that some additional flux beyond that due to diffusion crossed the plane $\eta=0$. This flux, he concluded, was proportional to the solute concentration gradient and therefore must be due to a dispersion mimicking the behavior of ordinary diffusion. He reached this conclusion by means of his considerable insight but provided little in the way of justification. Here, we reach the same conclusion, though by a means that is rigorous and based solely on the governing equations and boundary conditions. 
Equation (24) governing the function $g_{1}$ possesses closed-form solutions satisfying all required boundary conditions and integral constraints. For species transport and dispersion in a tube, the solution is

$$
\frac{4 g_{1}}{\lambda^{*}}=\frac{4 \lambda^{*} I_{0}\left(\kappa^{*} y^{*}\right)-\left(2 y^{* 2}+8 \lambda^{* 2}-1\right) I_{1}\left(\kappa^{*}\right)}{2 \lambda^{*} I_{1}\left(\kappa^{*}\right)-I_{0}\left(\kappa^{*}\right)}
$$

where $\kappa^{*}=1 / \lambda^{*}$. This result is plotted in Fig. 2 for a range of values of $\lambda^{*}$. The corresponding zerothorder eigenvalue for this problem is

$$
\frac{\alpha_{0}}{\lambda^{* 2}}=\frac{\left(3+16 \lambda^{* 2}\right) I_{1}^{2}\left(\kappa^{*}\right)-4 \lambda^{*} I_{0}\left(\kappa^{*}\right) I_{1}\left(\kappa^{*}\right)-2 I_{0}^{2}\left(\kappa^{*}\right)}{2\left[2 \lambda^{*} I_{1}\left(\kappa^{*}\right)-I_{0}\left(\kappa^{*}\right)\right]^{2}}
$$

This second result is equivalent to that obtained by McEldoon and Datta using integral methods for the case of no surface reactions [4]. Although this expression is valid over the full range of $\lambda^{*}$, it is difficult to evaluate numerically outside the range $0.1<\lambda^{*}<10$ due to the exponential growth of the Bessel functions when $\lambda^{*}$ is very small and due to indeterminacy of the expression when $\lambda^{*}$ is very large. To avoid these problems, we have developed a fit to this expression that is based on the asymptotic limits of Eq. (38) for both small and large $\lambda^{*}$. The result is

$$
\frac{4}{\alpha_{0}} \approx 192+\frac{4}{\lambda^{* 3 / 2}}+\frac{8}{\lambda^{* 2}}
$$

This approximation agrees with the results of Eq. (38) to within $3 \%$ over the full range of $\lambda^{*}$. Note that the common factor of four on the left of this expression is intentionally left in place since the Peclet number employed here is that based on the tube radius or channel half-height. Thus the appropriate expression for $\alpha_{0}$ for a Peclet number based on the diameter or full-height is obtained simply by replacing this four by a one. Also note that the limiting behavior of $\alpha_{0}=1 / 48$ for large $\lambda^{*}$ is the result obtained by Taylor for pressure-driven flow in a tube [3].

The results in Fig. 2 are useful in understanding the effects of nonuniform fluid velocity on the concentration field. Recall from Eq. (18) that the late-time concentration field is given by $c^{*} t^{* m / 2}=$ $f_{0}+\left(f_{1}+P e f_{0}^{\prime} g_{1}\right) / 2 \sqrt{t}+O[1 / t]$. Thus the function $g_{1}$ in the concentration field is multiplied by both the Peclet number and the derivative of $f_{0}$. For the case of a moving interface having a high concentration on the trailing side, the derivative of $f_{0}$ is everywhere negative. We thus see from Fig. 2 that the electroosmotic velocity profile increases the

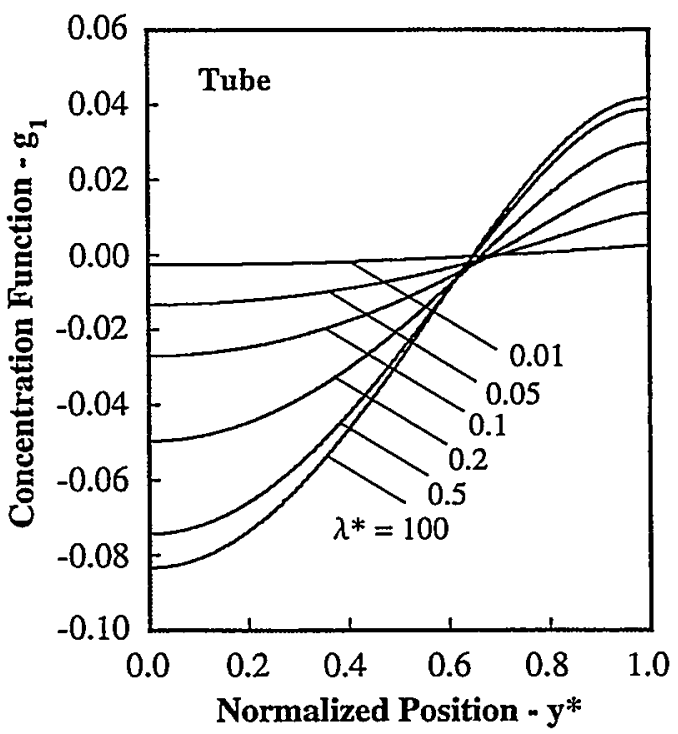

Figure 2. First-order correction describing the transverse variation in species concentration for electroosmotic flow in a tube.

concentration on the tube centerline, $y^{*}=0$, at all axial positions. Likewise, the concentration at the tube wall is everywhere reduced. For the case of an instantaneous source, however, the derivative of $f_{0}$ is positive for $\eta<0$ and negative for $\eta>0$. In this case, concentrations on the centerline are reduced behind the mid-plane of the solute peak, but are antisymmetrically increased on the centerline ahead. Concentrations at the tube walls exhibit just the opposite behavior.

The parallel results for electroosmotic flow in a channel are shown in Fig. 3 and are given by

$$
\frac{6 g_{1}}{\lambda^{*}}=\frac{6 \lambda^{*} \cosh \left(\kappa^{*} y^{*}\right)-\left(3 y^{* 2}+6 \lambda^{* 2}-1\right) \sinh \left(\kappa^{*}\right)}{\lambda^{*} \sinh \left(\kappa^{*}\right)-\cosh \left(\kappa^{*}\right)}
$$

and

$$
\frac{\alpha_{0}}{\lambda^{* 2}}=\frac{\left(2+12 \lambda^{* 2}\right) \sinh ^{2}\left(\kappa^{*}\right)-9 \lambda^{*} \sinh \left(\kappa^{*}\right) \cosh \left(\kappa^{*}\right)-3}{6\left[\lambda^{*} \sinh \left(\kappa^{*}\right)-\cosh \left(\kappa^{*}\right)\right]^{2}}
$$

This last result is again difficult to evaluate for very small or large values of $\lambda^{*}$. In this case, the fit based on asymptotic behavior for small and large $\lambda^{*}$ is

$$
\frac{4}{\alpha_{0}} \approx 210+\frac{5}{\lambda^{* 3 / 2}}+\frac{12}{\lambda^{* 2}}
$$

Again, this approximation agrees with the results of Eq. (41) to within 3\% over the entire range of $\lambda^{*}$. 


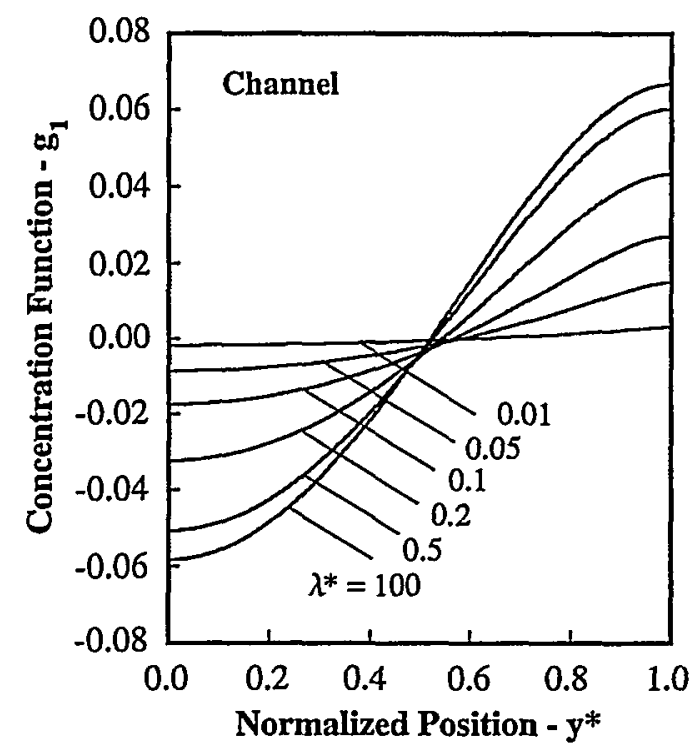

Figure 3. First-order correction describing the transverse variation in species concentration for electroosmotic flow in a channel.

Note that the limiting behavior of $\alpha_{0}=2 / 105$ for large $\lambda^{*}$ is the same as that obtained by Wooding for pressure-driven flow in a channel [5].

Figure 4 shows the dispersion coefficients for both the tube and channel as a function of the normalized Debye length. The solid curves shown are the approximate results for each case, while the symbols represent the exact solutions. The total height of the symbols represents a relative variation of about $8 \%$, so a symbol in contact with the curve indicates a relative error between the approximate and exact result of at most $4 \%$.

\section{MINIMUM DISPERSION}

The results above are useful in estimating the condition for minimum dispersion during transport over a fixed distance. This condition is equivalent to the condition of minimum theoretical plate height. The axial extent of spreading in a given time $t$ due to both diffusion and dispersion is given by

$$
\delta=2 \sqrt{D^{\prime} t}=2 \sqrt{D\left(1+\alpha_{0} P e^{2}\right) t}
$$

By expressing the time in terms of the travel distance $\ell, t=\ell / U=a \ell / P e D$ the extent of spreading

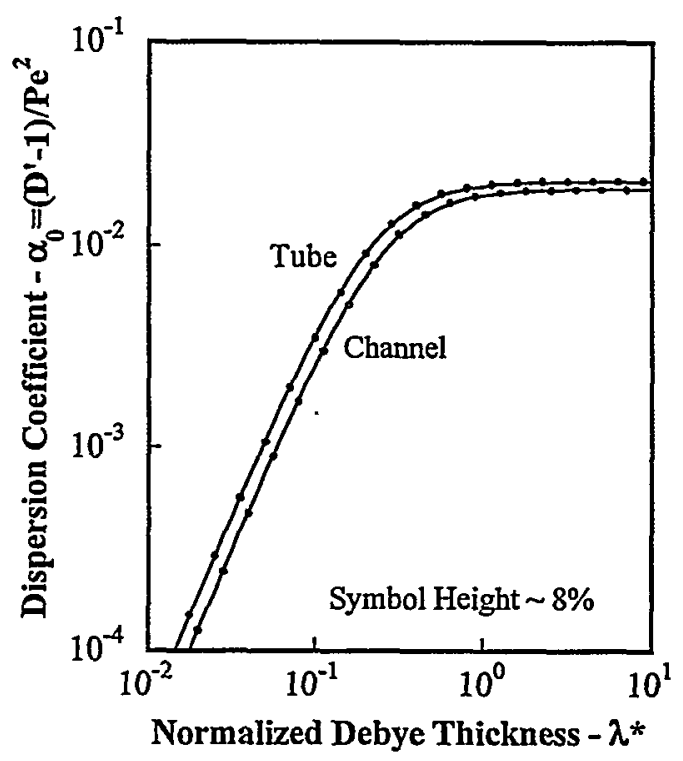

Figure 4. Dispersion coefficients as a function of Debye layer thickness. Symbols indicate exact solutions; lines indicate empirical fits.

may be rewritten as

$$
\delta=2 \sqrt{\frac{\left(1+\alpha_{0} P e^{2}\right) a \ell}{P e}}
$$

This expression exhibits a minimum given by the condition

$$
\frac{d \delta}{d P e} \rightarrow 0 \text { at } P e=\sqrt{\frac{1}{\alpha_{0}}}
$$

Note that this condition yields the Peclet number at which the spreading due to dispersion is just equal to that due to diffusion. The corresponding extent of spreading is

$$
\delta=2 \alpha_{0}^{1 / 4} \sqrt{2 a \ell}
$$

This is the minimum possible spreading of a sample transported over the fixed distance $\ell$.

In cases where the normalized Debye length is much less than unity, the asymptotic forms of Eqs. (39) and (42) may be used to evaluate the expressions above. For either the tube or channel, this asymptotic form shows that the coefficient of dispersion is proportional to the square of the normalized Debye length. In this limit, the optimum Peclet number is inversely proportional to the normalized Debye length and the extent of spreading 
over a fixed length of travel is independent of the tube or channel transverse dimension. The spreading is instead proportional to the square-root of the product of the Debye layer thickness and the distance of travel.

To illustrate the application of these results, consider the transport in a tube of $10 \mu \mathrm{m}$ radius and $100 \mathrm{~mm}$ length. Assuming a Debye layer thickness of $100 \mathrm{~nm}$, the normalized Debye length is $\lambda^{*}=10^{-2}$. From Eq. (39), this gives $\alpha_{0}=5 \times 10^{-5}$, and by Eq. (45) the optimum Peclet number is $P e=140$. For a diffusivity of $D=10^{-9} \mathrm{~m}^{2} / \mathrm{s}$, the equivalent mean fluid speed is $14 \mathrm{~mm} / \mathrm{s}$. This speed is a bit high for most electroosmotic flows, but it nevertheless illustrates the application these results and possible limitations of such application. Now from Eq. (46) we see that the extent of spreading over the $100 \mathrm{~mm}$ length of travel is just $0.24 \mathrm{~mm}$.

\section{SUMMARY}

Using analytical methods, we have determined the first-order concentration field and coefficient of axial dispersion of a neutral non-reacting solute in an incompressible electroosmotic flow. Here, in contrast to previous approaches, the dispersion is calculated by directly solving the governing transport equations. Using a late-time series to describe the full concentration field, the coefficient of dispersion arises naturally as a necessary condition for satisfying all required boundary conditions in transformed spatial and temporal coordinates.

Solutions based on the Debye-Hückel approximation are presented for both a circular tube and a channel of infinite width. These results recover the well-known solutions for dispersion in pressuredriven flows when the Debye length is very large. In this limit, the axial dispersion is proportional to the square of the Peclet number based on the characteristic transverse dimension of the tube or channel. In the limit of very small Debye lengths, we find that the dispersion varies as the square of the Peclet number based on the Debye length. Simple approximations to the dispersion as a function of the Debye length and Peclet number are also presented.

Although dispersion in both electroosmotic and pressure-driven flows grows as the square of the Peclet number, the coefficient of dispersion in an electroosmotic flow may be many orders of magnitude smaller than that for the parabolic velocity profile of a pressure-driven flow. The low coefficient of dispersion permits optimum operation of microchannel systems at very high Peclet num- bers. This minimizes the role of ordinary diffusion in electroosmotic flows, thus offering the potential for long-range transport with little axial spreading of solute peaks or interfaces due to either dispersion or diffusion.

In addition to providing physical insight into the nature of dispersion in electroosmotic flow, the analytical solutions presented here provide a valuable benchmark for developing numerical solutions to related problems. Direct numerical simulation of transport in electroosmotic flow is a challenging task because widely disparate length scales, spanning nearly seven orders of magnitude, must be resolved. Benchmark solutions are important in such cases since accurate numerical solutions are difficult to obtain.

\section{NOMENCLATURE}

$a$ tube radius or channel half-height

$c$ solute concentration

$c_{e}$ ion concentration

$D$ effective binary diffusivity

$D^{\prime}$ effective diffusivity inclusive of dispersion

$E_{x}$ applied axial electric field: $E_{x}=-d \phi / d x$

$f_{k}$ axial concentration functions

$F$ Faraday constant

$g_{j}$ transverse concentration functions

$m$ interface $(m=0)$ or plane source $(m=1)$

$n$ channel $(n=0)$ or tube $(n=1)$

$P e$ Peclet number: $P e=U a / D$

$R$ ideal gas constant

$t$ time

$T$ temperature

$u$ local fluid speed

u local fluid velocity

$U$ mean fluid speed

$x$ axial position

$y$ transverse position

$z$ charge number

$\alpha_{k}$ eigenvalues

$\epsilon \quad$ dielectric constant

$\mu \quad$ kinematic viscosity

$\lambda$ Debye length

$\rho_{e}$ charge density

$\phi \quad$ electric potential

$\zeta \quad$ surface electric potential

Normalized Variables

$\mathrm{E}^{*}$ electric field: $\mathrm{E}^{*}=-\nabla \phi / E_{x}$

$t^{*}$ time: $t^{*}=D t / a^{2}$

$u^{*}$ local fluid speed: $u^{*}=u / U$

$x^{*}$ axial position: $x^{*}=(x-U) / a$ 
$y^{*} \quad$ transverse position: $y^{*}=y / a$

$\eta$ axial position: $\eta=x^{*} / 2 \sqrt{t^{*}}$

$\lambda^{*}$ Debye length: $\lambda^{*}=\lambda / a$

$\kappa^{*}$ inverse Debye length: $\kappa^{*}=1 / \lambda^{*}$

$\zeta^{*}$ surface potential: $\zeta^{*}=z F \zeta / R T$

Subscripts and Superscripts

$j$ order of solution

* asterisk denotes normalized variable

- hat denotes initial distribution

\section{REFERENCES}

1. R. F. Probstein, Physicochemical Hydrodynamics, John Wiley \& Sons, New York, NY (1995).

2. C. L. Rice and R. Whitehead, "Electrokinetic Flow in a Narrow Cylindrical Capillary," J. Phys. Chem., 69, 4017, 186-203, 1965.

3. G. I. Tayor, "Dispersion of Soluble Matter in Solvent Flowing Slowly Through a Pipe," Proc. Roy. Soc. Lond., A 219, 186, 1953.

4. J. P. McEldoon and B. Datta, "Analytical Solution for Dispersion in Capillary Chromatography with Electroosmotic Flow," Anal. Chem., 64, 227, 1992.

5. R. A. Wooding, "Instability of a Viscous Liquid of Variable Density in a Vertical Hele-Shaw Cell," J. Fluid Mech., 7, 501, 1960.

\section{ACKNOWLEDGMENT}

The authors wish to thank Dr. G. H. Evans for his careful review of this paper. This work was funded by a Sandia Phenomenological Modeling and Engineering Simulations LDRD. Sandia is operated by Sandia Corporation for the United States Department of Energy.

\section{APPENDIX}

Two subtle manipulations are required in solving the governing equations presented here. The purpose of this appendix is to provide some additional guidance in these subtleties.

The first subtlety arises in obtaining the $f_{1}$ solution for the case of a translating interface. In this case, the governing equation (28) possesses two homogeneous solutions, $f_{1 h}$, one of the form $e^{-\eta^{\prime 2}}$ and the other of the form $e^{-\eta^{\prime 2}}$ erfi $\eta^{\prime}$ where erfi $z=$ $-i \operatorname{erf} i z$ is the imaginary error function. Both of these automatically satisfy the required conditions $f_{1 h} \rightarrow 0$ as $\eta \rightarrow \pm \infty$, so the unknown constants multiplying these two solutions cannot be determined from the boundary conditions. The only remaining condition is the integral constraint of the initial condition given by Eq. (20) for $p=0$. The second solution is odd about the origin, so its integral over the axial domain vanishes automatically. It thus appears that the constant multiplying this second solution cannot be determined from any of the prescribed conditions. Upon examination, however, we find that the second homogeneous solution exhibits the behavior $f_{1 h} \rightarrow 1 / \eta^{\prime}$ as $\eta \rightarrow \pm \infty$. As a result, the integral of $e^{-\eta^{\prime 2}}$ erfi $\eta^{\prime}$ over either half-space is infinite. This homogeneous solution thus represents an infinite total solute in the right half-space and a corresponding infinite total solute deficit in the left half-space. The solution is therefore aphysical and so is appropriately discarded by taking the unknown constant to be zero. The constant multiplying the first homogeneous solution is then determined by the $p=0$ moment, yielding the 1 that appears in the numerator of Eq. (36).

The second subtlety arises in the corresponding $f_{1}$ solution for the problem of a plane source. The governing equation (28) again possesses two homogeneous solutions, in this case one of the form $\eta^{\prime} e^{-\eta^{\prime 2}}$ and the other of the form $1-\eta^{\prime 2} e^{-\eta^{\prime 2}}$ erfi $\eta^{\prime}$. The first of these is odd with respect to the origin, while the second is even. This second solution automatically satisfies the prescribed boundary conditions and the initial condition given by Eq. (20) for $p=0$. Since this function is even, it also automatically satisfies the required initial condition for $p=1$. The unknown constant multiplying this solutions thus appears to be indeterminate. In this case, however, we find that the magnitude of the first moment of the solution is infinite over each half-space. This is again not physically possible, and so this second homogeneous solution is discarded by taking the undetermined constant to be zero. The constant multiplying the first homogeneous solution is then determined by the first moment, giving rise to the 3 appearing in Eq. (36). 
UNLIMITED RELEASE

INITIAL DISTRIBUTION

K. F. Jensen

Massachusetts Institute of Technology

Building 6-469

Chemical Engineering

Cambridge, MA 02139

1413 T. E. Michalske, 1114

1425 D. R. Adkins, 1715

1425 G. C. Frye-Mason, 1715

0710 A. P. Sylwester, 1845

0149 C. E. Meyers, 4000

1094 M. C. OBorny, 7524

9001 M. E. John, 8000

Attn: R. C. Wayne, 8400

D. L. Crawford, 8900

9056 J. Vitko, 8100

9671 J. S. Schoeniger, 8120

9214 C. F. Melius, 8130

9403 J. M. Hruby, 8230

9054 W. J. McLean, 8300

Attn: D. R. Hardesty, 8361

F. P. Tully, 8353

R. W. Carling, 8362

9041 J. S. Binkley, 8345

9042 S. K. Griffiths, 8345 (5)

9042 R. H. Nilson, 8345 (5)

9671 E. B. Cummings, 8351

9056 P. H. Paul, 8351

9056 L. A. Rahn, 8351

9671 D. W. Arnold, 8358

9671 C. G. Bailey, 8358

9161 W. Bauer, 8358

9671 D. J. Rakestraw, 8358

9405 T. M. Dyer, 8700

Attn: M. I. Baskes, 8712

G. J. Thomas, 8715

J. C. F. Wang, 8713

K. L. Wilson, 8716

S. M. Foiles, 8717

9402 C. M. Hartwig, 8701

9003 D. L. Lindner, 8902

9214 R. C. Armstrong, 8980

0841 P. J. Hommert, 9100

0826 W. L. Hermina, 9111

0826 D. R. Noble, 9111

0826 P. R. Schunk, 9111

0843 A. C. Ratzel, 9112

0827 C. C. Wong, 9114

1003 B. L. Spletzer, 9611
9018 Central Technical Files, 8940-2 (3)

0899 Technical Library, 4916

9021 Technical Communications Dept., 8815/ Technical Library, MS 0899, 4916

9021 Technical Communications Dept., 8815 